\title{
Development of a Sensor Module for Estimating the Center of Pressure in Low Cost Gait Rehabilitation Robots
}

\author{
Su-Hong Eom*, Ki-Sun Song, Shin-Yoon Kang, Eung-Hyuk Lee \\ Department of Electronic Engineering Korea Polytechnic University, \#2121, Jeongwang-Dong, Siheung-City, \\ Gyeonggi-Do, Republic of Korea.
}

* Corresponding author. Email: sheom@kpu.ac.kr

Manuscript submitted August 11, 2014; accepted June 8, 2015.

doi: 10.17706/jsw.10.6.767-774

\begin{abstract}
Gait information of pedestrians is an important factor in gait rehabilitation. The most important factor is to measure the center of pressure (CoP) compared to other measurements because gait patterns are varied according to CoP. Multiple strain gauges, multi-axis load cells, and pneumatic tube sensors have been used to measure the $\mathrm{CoP}$ of the body. Because these sensors are large sizes and high prices, these sensors are not suitable for low cost gait rehabilitation robots. Thus, in this study a sensor module that can estimate the $\mathrm{CoP}$ of the body roughly is proposed. The proposed sensor module uses resistance variable sensors, which are able to detect positions. For applying the proposed sensor, an extra device is proposed and is combined with sensors for configuring a module. A comparison test is implemented using a pressure sensor with an FSR array structure in order to verify the proposed method. In results, the proposed method represents an agreement in directivity more than $93 \%$.
\end{abstract}

Key words: Slope directions, prosthesis, walking rehabilitation, sensor module for CoP, CoP.

\section{Introduction}

The elderly represents physical functions due to aged body functions compared to that of young people [1]. Functional differences are delaying body response time, decreasing cognitive functions, decreasing the sense of equilibrium, and so on [2]. These functional differences cause limitations in the gait of the elderly such as gait speed, step length, gait frequency, range of motion, decreasing angular velocity, and decreasing the sense of equilibrium [1].

Abnormal gaits in the elderly become an important factor of inhibiting ADL (Activities of Daily Living) and that leads to a passive attitude in social participations [3].

Thus, the gait rehabilitation of the elderly is required for improving their quality of life (QOL).

Systematic and repetitive rehabilitation exercises for the elderly are needed to maintain and improve their muscle power in order to rehabilitate gait functions [4].

Such repetitive exercises can be performed using robots. Because robots can easily perform repetitive actions and assist postures of trainees, it is possible to expect ensuring safeties and high training effects. As effects of using practical robots in rehabilitation on neurorehabilitation therapies have been increased, it receives much attention in rehabilitation medicine [5].

Gait rehabilitation using robots is divided into two different categories. First, it uses a threadmill based robot, Locomat by Hocoma, for patients without self-support capabilities. Second, it uses a knee brace for patients who have self-support capabilities but require gait exercises. The knee brace is classified into 
manual and electric types. The BIONIC LEG by AlterG is one of electric types.

Gait rehabilitation devices using robot technologies collect and analyze the gait information of trainees through various sensors for improving rehabilitation performances. The information to be collected and analyzed are gait speeds, gait patterns, changes of CoP (center of pressure), knee angles, ankle angles, and so on. The most important factor is CoP compared to other elements because gait patterns are varied according to $\mathrm{CoP}$ and that leads to change knee and ankle angles [6].

For measuring CoP, multiple strain gauges, multi-axis load cells, and pneumatic tubes are currently used 7]-[9]. However, these sensors are large sizes and high prices. Thus, in some systems a force sensing resistor (FSR), which is a film type pressure sensor, is used to roughly estimate CoP [10]. Although the CoP estimation method using FSR is low cost relatively, it is possible to front and rear CoP of the foot but is difficult to estimate left and right CoP of the foot.

Therefore, in this study a method that compensates the disadvantage in the low cost FSR method is proposed as follows. The proposed method applies resistance variable sensors according to positions. By attaching the sensor to the heel of toe, the positions are detected according to weight drifting in order to estimate CoP roughly. In the case of the simple position of the sensor, it decreases the reliability of the obtained data because several points are detected due to the sole area of the foot. Thus, in this study a device that applies pressures to sensors through dividing the sole area of the foot into several sections is proposed and is to be modularized

\section{Method}

In the general measurement method of CoP, it is estimated using a multi-axis load cell by comparing pressure values applied to each axis and is determined by the large pressure. In addition, multiple strain gauges are attached to the divided sections of the sole of the foot. Then, CoP can be estimated by a section with the largest change in outputs. In the case of the method using pneumatic tubes and multiple FSRs, it uses the similar process to that of the strain gauge.

However, there are some problems in using these estimation methods. Regarding the method using load cells, the sensors are expensive and large volumes. In the case of using strain gauges, pneumatic tubes, and FSRs, the sensors are to be located according to divided sections.

Thus, in this study an estimation method of CoP using a single and low cost sensor is proposed as follows.

\subsection{Proposed Device for Estimating CoP}

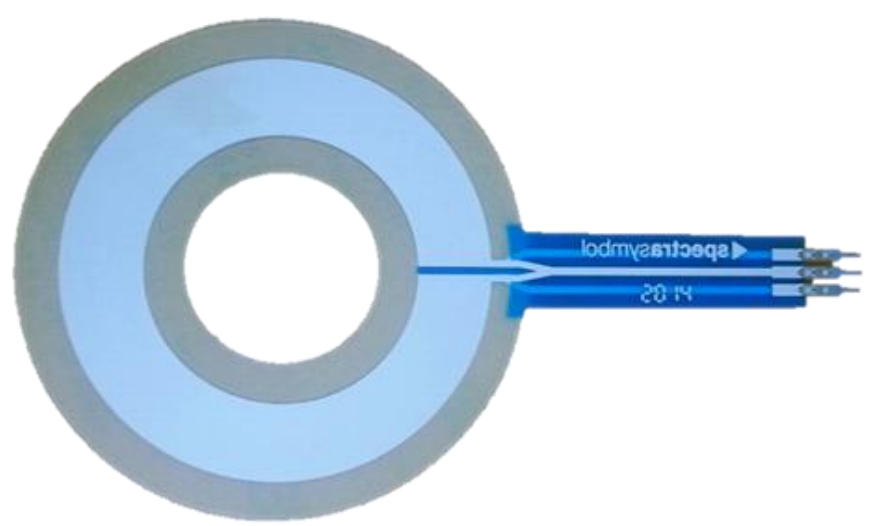

Fig. 1. Resistance variable sensor according to positions.

The proposed method uses a resistance variable sensor according to positions. Recently resistance variable sensors have been used in various industrial devices and a representative application of the sensor 
is equalizer frequency sliders in audio systems. The position detection sensor used in this study is not a linear type conventionally used in frequency sliders but a circular type as shown in Fig. 1.

As it is a circular type, it is possible to detect weight drifts by attaching it to either the heel or toe at the sole of the foot. The sole of the foot, however, detects information as a wide area because the sole of the food is a planar area. In the case of the resistance variable sensor for detecting positions, it decreases resolutions significantly as pressures are applied to a wide area. Thus, the following method is proposed for improving the precision of sensing the sensor from the wide area. Fig. 2 shows the kinematic structure of the proposed device.

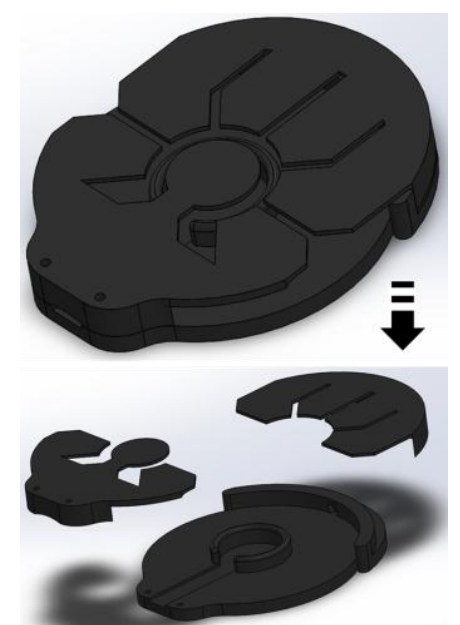

Fig. 2. The kinematic structure of the proposed device.

The wide sole of the foot is divided into six different directions and sensor inputs are applied to each section. A sensor is inserted to the center of the device for measuring the ground reaction force during the gait exercise.

\subsection{Estimating CoP Using the Resistance Variable Sensor According to Positions}

In the case of the general resistance variable sensors, it measures the change in resistance values by converting it to voltage values based on the voltage division rule as shown in Eq. (1). As the sensor used in this study is a resistance variable sensor, it obtains the output value using the same method. Also, the sensor increases resistance values according to pushing the sensing points along the clockwise direction(see Fig. 3 ). Then, the CoP can be estimated by calculating the output values at both left and right points as angle values as noted in Eq. (2). The reason that the maximum angle value is not determined as $360^{\circ}$ in Eq. (2) is due to the consideration of the characteristics of the sensors. It will be described in the following experiment.

$$
\begin{gathered}
V_{\text {out }}=\frac{R_{\text {ref }}}{R_{\text {Sensor }}} \times V_{\text {in }} \\
\text { Angle }=\left(\frac{360}{R_{\text {Sensor_Max }}}\right) \times R_{\text {Measured_value }}
\end{gathered}
$$

\subsection{Sensor Data Linearization Method}

The output format of sensor is classified to digital and analog. Digital type is for reading sensor data by communication methods. Analog type is normally variable resistance type. However, there are two cases of resistance variation width by characteristics of sensor, one is linear variation and the other is non-linear variation. Non-linear variation output is applied various linearization methods : linearization to dividing interval, estimation of trend line by log curve, estimation methods by Multi-dimensional equation, etc.

But then, the output data which seems linearly is applied relatively simple methods : average to dividing 
interval, linearization based on slope of data, etc.

In this paper, the sensor which applied is variable resistance type sensor that seems linearity. therefore, we applied the least-squares method for linearization of output data. The linearized data is applied to angle calculation equation for obtain formalized data. the generalization equation of least-squares method is following Eq. (3).

$$
\sum\left(y_{i}-\left(a x_{i}+b\right)\right)^{2}
$$

\section{Results and Discussion}

An experiment was performed for verifying the proposed method. In the experiment, a module was fabricated as shown in Fig. 4. The fabricated module was configured three different sections. As shown Fig. 4, Section 1 is used to attach the sensor, Section 2 is used to push the pressure sensor, and Section 3 is used to push the resistance variable sensor. The sections to be pushed are fabricated with silicon. The sensor which applied in this paper is sparkfun's SEN-08678 sensor.

For verifying the performance of the fabricated sensor, the subject section was divided into six sections for detecting positions. Table 1 shows the results. In addition, the data in which pressures are applied to more than two sections simultaneously in the sole of the foot was obtained. The results are presented in Table 2 and Table 3. Considering Table 1 and Table 2, it was verified that the detection of the proposed position is precisely performed for a single input.

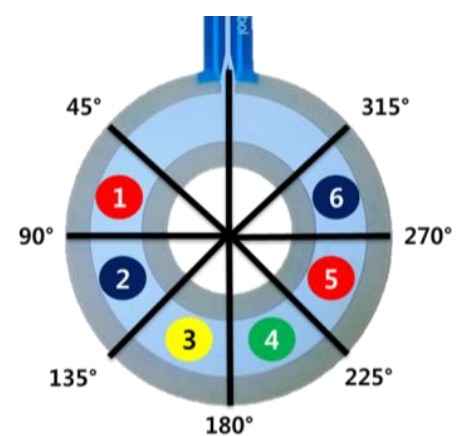

Fig. 3. Partitions of sensor.
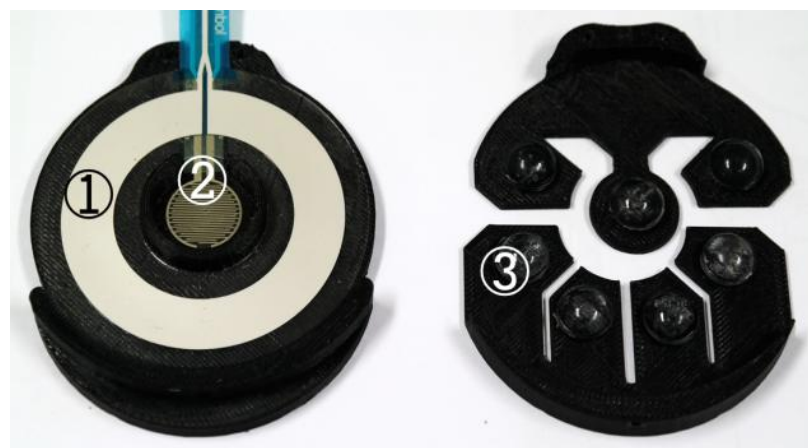

Fig. 4. Fabricated module.

Table 1. Value of Six Sections for Detected Positions

\begin{tabular}{|c|c|l|}
\hline \multirow{2}{*}{} & \multicolumn{2}{|c|}{ Measure value of Sensor } \\
\cline { 2 - 3 } & Volt value of Position & Degree value of Position \\
\hline 1 & $0.92 \mathrm{~V}$ (Position 1) & 66 。 \\
\hline 2 & $1.52 \mathrm{~V}$ (Position 2) & 110 。 \\
\hline 3 & $2.20 \mathrm{~V}$ (Position 3) & 159 。 \\
\hline 4 & $2.80 \mathrm{~V}$ (Position 4) & $202 。$ \\
\hline 5 & $3.41 \mathrm{~V}$ (Position 5) & $247 。$ \\
\hline 6 & $4.05 \mathrm{~V}$ (Position 6) & 393 。 \\
\hline
\end{tabular}

More than two section, however, the sections less than $30^{\circ}$ and more than $330^{\circ}$ showed decreases in precision and there were errors in the section more than $330^{\circ}$ as pressures were applied together with other sections simultaneously. Thus, in this study these sections were excluded in order to prevent applying pressures to the sections in fabricating the device. 
Table 2. Measured of the Data in Two Sections

\begin{tabular}{|c|c|c|c|c|c|}
\hline & \multicolumn{5}{|c|}{ Measure value of Sensor } \\
\hline Posi-tins & 1 and 2 & 2 and 3 & 3 and 4 & 4 and 5 & 5 and 6 \\
\hline V & $0.68 \mathrm{~V}$ & $1.03 \mathrm{~V}$ & $1.45 \mathrm{~V}$ & $1.73 \mathrm{~V}$ & $2.11 \mathrm{~V}$ \\
\hline Deg & 83 。 & 125 。 & 175 。 & 207 。 & $255 。$ \\
\hline Posi-tins & 1 and 3 & 2 and 4 & 3 and 5 & 4 and 6 & - \\
\hline V & $0.72 \mathrm{~V}$ & $1.20 \mathrm{~V}$ & $1.70 \mathrm{~V}$ & $2.10 \mathrm{~V}$ & - \\
\hline Deg & $86 。$ & 145 。 & 205 。 & $252 。$ & - \\
\hline Posi-tins & 1 and 4 & 2 and 5 & 3 and 6 & - & - \\
\hline V & $0.86 \mathrm{~V}$ & $1.46 \mathrm{~V}$ & $2.06 \mathrm{~V}$ & - & - \\
\hline Deg & 103。 & $175 。$ & 250 。 & - & - \\
\hline Posi-tins & 1and 5 & 2 and 6 & - & - & - \\
\hline V & $1.07 \mathrm{~V}$ & $1.86 \mathrm{~V}$ & - & - & - \\
\hline Deg & $130 。$ & $223 。$ & - & - & - \\
\hline Posi-tins & 1 and 6 & - & - & - & - \\
\hline V & $1.48 \mathrm{~V}$ & - & - & - & - \\
\hline Deg & $178 。$ & - & - & - & - \\
\hline
\end{tabular}

Table 3. Measured of the Data to more than Two Sections

\begin{tabular}{|c|c|c|c|c|}
\hline \multirow[b]{2}{*}{ Positions } & \multicolumn{4}{|c|}{ Measure value of Sensor } \\
\hline & $1 \sim 6$ & $2 \sim 6$ & $3 \sim 6$ & $4 \sim 6$ \\
\hline V & $1.48 \mathrm{~V}$ & $1.83 \mathrm{~V}$ & $2.02 \mathrm{~V}$ & $2.08 \mathrm{~V}$ \\
\hline Deg & 178 。 & $220 。$ & $245 。$ & 250。 \\
\hline Positions & $1 \sim 5$ & $2 \sim 5$ & $3 \sim 5$ & - \\
\hline V & $1.09 \mathrm{~V}$ & $1.45 \mathrm{~V}$ & $1.70 \mathrm{~V}$ & - \\
\hline Deg & $130 。$ & $175 。$ & $204 。$ & - \\
\hline Positions & $1 \sim 4$ & $2 \sim 4$ & - & - \\
\hline V & $0.88 \mathrm{~V}$ & $1.20 \mathrm{~V}$ & - & - \\
\hline Deg & $106 。$ & 145。 & - & - \\
\hline Positions & $1 \sim 3$ & - & - & - \\
\hline V & $0.73 \mathrm{~V}$ & - & - & - \\
\hline Deg & 88 。 & - & - & - \\
\hline
\end{tabular}

For verifying the reliability of the proposed module, a gait experiment was performed under the environment as shown in Fig. 5. The gait sections are divided into flat/lateral tracks. Fig. 6-Fig. 8 show the results of this gait experiment.

In the case of the flat track, changes in weight movements to left/right directions were detected as the gait was implemented from the stance to the swing phases.

In Fig. 6, The variation of CoP Data is changed from point_A to point_B. It is analyzed to movement of CoP, from swing phase to stance phase.

It is a characteristic presented in gait patterns. Also, it is verified that the proposed module was effective to estimate CoP. In the case of the lateral track, as illustrated in Fig. 7. A, B and C the weight drifts to the slope directions were detected. 


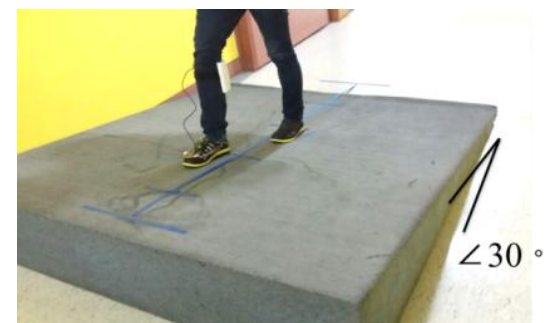

Fig. 5. Experiment environment.

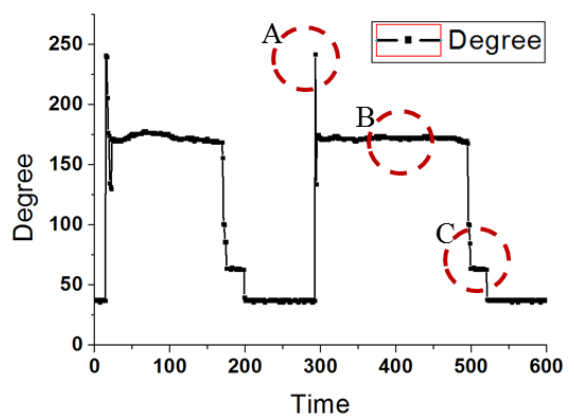

Fig. 7. The graph of walking of lateral track (Left->Right). Fig. 8. The graph of walking of lateral track

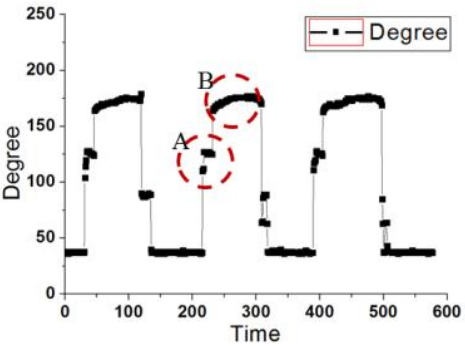

Fig. 6. The graph of walking of flat track

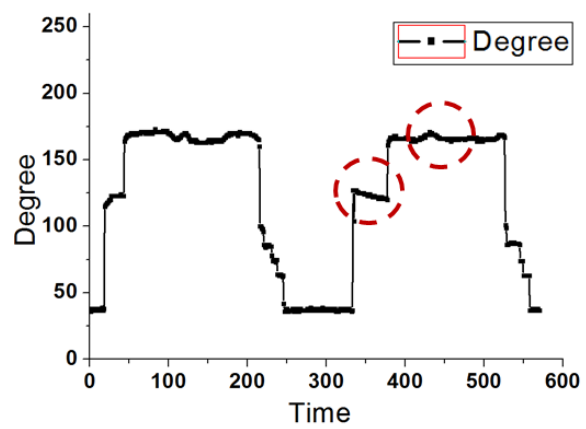

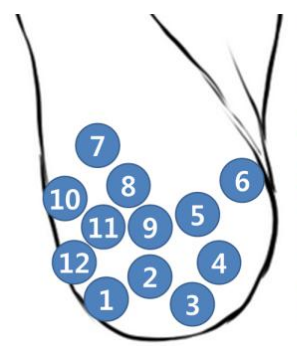

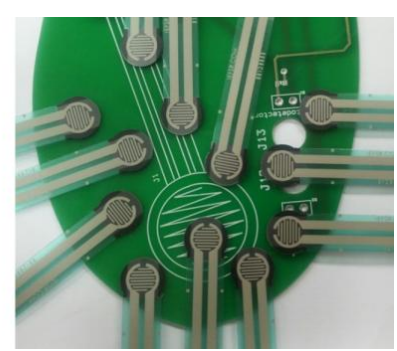

(Right->Left).

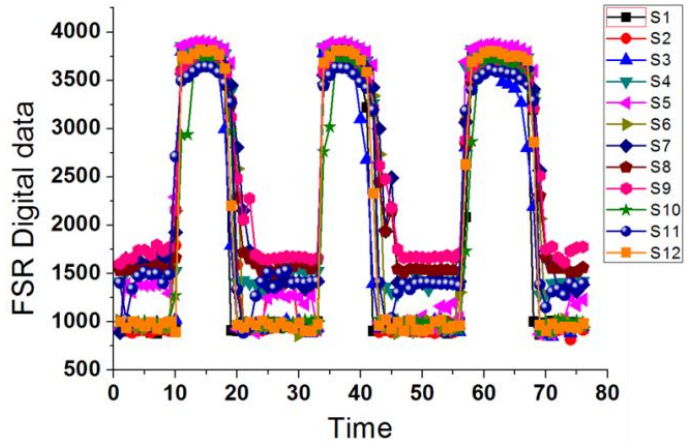

Fig. 9. FSRs were installed to the sole of the foot as an array type. Fig. 10. The graph of walking of flat track (FSRs).

In the interval of lateral, the case of movement of CoP from right to left, there seems to same pattern to the case of movement of CoP in flat track. The reasons for that are following : In case of lateral track, The lateral angle of ankles is variations by the walking directions of the human body. It is in case that ankle is bent to inward of body. there are difficult problems due to the structure of human body, because it is for measurement of directionality of CoP. But then, in case of measurement in the form of a fixed ankle such as prosthesis, the data of $\mathrm{CoP}$ is measured correctly in lateral track.

For ensuring the preciseness of the experiment, FSRs were installed to the sole of the foot as an array type in order to measure pressure distributions (see Fig. 9). Then, the measurements were used to implement comparative experiments in which the comparison was only implemented for directivity only because the method proposed in this study was a directivity based CoP estimation method. The Fig. 10 is graph which measured in flat track, based on Fig. 9. in this graph, S10 is increase slow than S4, S5. And, S3 is decrease fast than $\mathrm{S} 10$. it is analyzed that CoP is moved from swing phase to stance phase.

In the comparison results, the proposed module, and the FSR array sensor, the CoP represented an agreement rate of $93 \%$.

\section{Conclusion}


In this study a module that estimates CoP in gait exercises using low cost sensors was proposed. The proposed method used resistance variable sensors according to positions. For obtaining the reliable data of the subject sensors, a proper device was proposed. Gait experiments were implemented for flat/lateral tracts in order to verify the proposed module. In results, it was possible to estimate CoP in all these three tracts. In addition, for ensuring the reliability of the detected data FSRs were installed to the sole of the foot as an array type for measuring pressure distributions and the results were compared with the pressure distributions. In the results of this comparison, it showed an agreement of $93 \%$ in its directivity.

Based on the results, it is considered that the proposed method is useful to estimate CoP for low cost gait rehabilitation robots in future.

In this study, however, it is not possible to obtain a pressure distribution like load cells because the objective is to develop a low cost robot. Therefore, it is necessary to merge it into other sensors for improving such a problem.

\section{Acknowledgment}

"This research was supported by the MSIP(Ministry of Science, ICT\&Future Planning), Korea, under the C-ITRC(Convergence Information Technology Research Center) support program (NIPA2014-H0401-14-1003) supervised by the NIPA(National IT Industry Promotion Agency)"

\section{References}

[1] Karlijn, F. D. L., Anil, M. T., \& Frank, E. D. L. (2011). Loss of White Matter Integrity is Associated with Gait Disorders in Cerebral Small Vessel Disease, 34(1), 73-87.

[2] Diana, B. W., Longstreth, W. T., Mary, F. L., \& Annette, L. F. (2010). Cognition and the risk of hospitalization for serious falls in the elderly: Results from the cardiovascular health study. Journals of Gerontology Series A: Biomedical Sciences and Medical Sciences, 65(11), 1242-1249.

[3] Minoru, Y., Hidenori, A., Kazuki, U., Shuhei, M., Koutatsu, N., Buichi, T., Yusuke, T., Mamoru, I., \& Tomoki, A. (2011). Effect of resistance training on physical performance and fear of falling in elderly with different levels of physical well-being. Age and Ageing, 40(5), 637-641.

[4] Furui, W., Duygun, E. B., \& Nilanjan, S., Impact of visual error augmentation when integrated With assist-as-needed training method in robot-assisted rehabilitation. Transactions on Neural Systems And Rehabilitation Engineering, 18(5).

[5] Krebs, H. I., Volpe, B. T., Aisen, M. L., \& Hogan, N. (2000). Increasing productivity and quality of care: Robot-aided neuro-rehabilitation. Journal of Rehabilitation Research and Development, 37(6), 639-652.

[6] Lawson, B. E., Shultz, A. H., \& Goldfarb, M. (2013). Evaluation of a coordinated control system for a pair of powered transfemoral prostheses. Proceedings of the IEEE International Conference on Robotics and Automation.

[7] Sup, F. (2009). A Powered Self-Contained Knee and Ankle Prosthesis for Near Normal Gait in Transfemoral Amputees, Nashville, Tennessee, Vanderbilt University, 2009.

[8] Gregg, R. D., Lenzi, T., Fey, N. P., Hargrove, L. J., \& Sensinger, J. W. (2013). Experimental effective shape control of a powered transfemoral prosthesis. Proceedings of the IEEE International Conference on Rehabilitation Robotics.

[9] Kawamoto, H., Hayashi, T., Sakurai, T., Eguchi, K., \& Sankai, Y. (2009). Development of single leg version of HAL for hemiplegia. Proceedings of the 31st Annual International Conference of the IEEE EMBS.

[10] Su, H. E., Mun, S. J., Eung, H. L., \& Pyung, S. K. (2014). A method for estimating joint angles value of knee joint rehabilitation robot. ICIC Express Letters, 8(2). 
Su-Hong Eom was born in Korea, in 1984. He received his B.E., M.E. degree in the Department of Information and Communication Engineering from Korea Polytechnic University. Now, he is a student in $5^{\text {rd }}$ Semester, Korea Polytechnic Technology University, Department of Information and Communication Engineering $\mathrm{PhD}$. His research interest intelligent robotics and control, rehabilitation engineering.

Ki-Sun Song was born in Korea, in 1989. He received his B.E. degree in the Department of Electronics engineering from Silla University. Now, he is a student in $3^{\text {rd }}$ Semester, Department of Electronics Engineering from Korea Polytechnic University M.E. His research interests rehabilitation robotics and human interface, rehabilitation engineering.

Sin-Yoon Kang was born in Korea, in 1987. He received his B.E. degree in the Department of Electronics engineering from Korea Polytechnic university. Now, he is a student in $3^{\text {rd }}$ Semesters Department of Electronics Engineering from Korea Polytechnic University M.E. he research interests intelligent robotics and control, rehabilitation engineering.

Eung-Hyuk Lee war born in Korea, in 1962. He received his B.E. degree in the Department of Electronics Engineering from INHA University. And he received his M.E., PhD degree in the Department of Information Engineering. Now, he is a professor of the Department of Electronics Engineering Korea Polytechnic University. His research interest are intelligent service robotics and control, mobile health care system and rehabilitation engineering. 